Original Paper

\title{
A Study on Impact of Pellets on Different Targets- A Preliminary Study
}

\author{
Devika G $^{1}$, S.D. Dhaarani ${ }^{1}$, Sona Ponnuswamy ${ }^{1}$, Manashree Mane ${ }^{2}$ \\ ${ }^{1}$ BSc Student, ${ }^{2}$ Assistant Professor, Department of Forensic Science, JAIN (Deemed-to-be University), Bangalore, \\ Karnataka, India
}

\begin{abstract}
Background: Forensic investigators often comes across cases where the projectile is found in severely damaged condition, fragmented, or not recovered from the victim or the scene of crime. Analysis of entry and exit hole becomes essential to determine the calibre or type of projectile used to aid investigation.

Purpose: The present study was performed to study impact made by lead and copper pellets fired from Hammerli AR 20 7.5 Joules where targets used were fabric (cotton and silk), glass (sodalime glass, textured and privacy pane) and wood (MDF and plywood).

Procedure: The current study was performed using fabric (cotton and silk), glass (sodalime glass, textured and privacy pane) and wood (MDF and plywood) by firing pellets firing at10 metres.

Main findings: The fibres protruded outwards around margin of entry hole. The diameter of entry hole, exit hole and penetration depth was consistent among the same type of target.

Conclusion: Post analysis of the present data was done to study the influence of lead and copper pellets on wood, cloth and glass on entry and exit holes. However, it was found that in both cases (i.e., entry and exit holes), the target groups have a significant difference but in case of pellets of same calibre, there is an insignificant difference with entry and exit holes.
\end{abstract}

Keywords: Targets; airgun; entry and exit hole; pellets.

\section{Introduction}

"Firearms" means arms of any description designed or adapted to discharge a projectile or projectiles of any kind by the action of any explosive or other forms of energy, and includes:

\section{Corresponding Author:}

\section{Ms. Manashree Mane}

Assistant Professor Department of Forensic Science, JAIN (Deemed-to-be University), Bangalore- 560027, Karnataka, India, Email address: manashree4n6@ gmail.com, Mobile No: +919769140 575
- artillery, hand-grenades, riot-pistols, or weapons of any kind designed or adapted for the discharge of any noxious liquid, gas, or other such things,

- accessories for any such firearm designed or adapted to diminish the noise or flash caused by the firing thereof,

- parts of, and machinery for manufacturing, firearms, and

- carriages, platforms, and appliances for mounting, transporting, and serving artillery; ${ }^{[1]}$ 
Firearms consist of a long tube-like structure termed as barrel, which is closed at the back end called the breech end and the front open end called as the muzzle end. When the trigger is pulled, the sear is disengaged from the bent, hammer moves forward and strikes a small pushrod (firing pin or striker). Assault rifles are automatic weapons firing intermediate rifle bullets having a detachable magazine of 20 rounds or more. In air rifle and air pistol, compressed air is used to fire lead slugs. Improvised firearms or country made firearms are common in India.

The size and velocity of the bullet are the two most important factors that affect the wounding power. Another factor which affects the wounding is the density of the tissue with greater destruction in dense tissue like bones than the soft tissues. A bullet of smaller calibre than that of the weapon may cause slight bruising ${ }^{[2]}$.

Due to air resistance and gravitational pull of the earth, the bullet follows a downward curved path or trajectory when it leaves the barrel. The path followed by the projectile can be determined by knowing the various aspects such muzzle velocity, the angle of elevation of the barrel, sectional density of the bullet and the bullet shape ${ }^{[3]}$. According to the type of target surfaces, the penetrative properties of a projectile differ. The incidence velocity, bullet construction, target surface construction comes into picture when a projectile hits target material that is hard. A high velocity projectile is capable of even penetrating through the hard target surfaces made of hardened steel. On the other hand, shotguns have relatively low penetration capability and cause considerable damage. However, these firearms which has individual pellets, spread, and cause great injuries. The severity on human body depends upon the shape of the projectile rather than its kinetic energy [4]. As target strength increases, the penetration depth of projectile decreases. Therefore, cavitation threshold increases with target strength ${ }^{[5]}$.

Gelatin blocks are helpful in wound ballistics study wherein researchers use for designing ammunition, ballistic protective equipment which includes body armour. Use of gelatin blocks simulates the damage seen in soft tissues. Usually, $10 \%$ or $20 \%$ gelatin blocks are conditioned at $4-10^{\circ} \mathrm{C}$. Bullets do not tend to deform greatly during soft tissues impact and results in complete perforation of targets. Bullets that interact with harder surfaces like body armour, may deform or fragment. If the bullet remains in the body after impact, the kinetic energy gets transferred to the tissues ${ }^{[6]}$.

The projectile at various instances hits intermediary targets such as cloth, glass or wood. Natural fibers are wholly derived from animal or plant sources. The wide use of undyed white cotton fibers for different purpose has made its evidential value almost meaningless, although dyed cotton, in some cases, has enhanced its evidential significance ${ }^{[7]}$. The normal window glass when hit by a projectile produces radiating lines commonly known as radial fractures, and the circular lines termed as concentric fractures. The $3 \mathrm{R}$ rule becomes difficult to be applicable in absence of radial or concentric fracture lines such as broken tempered glass ${ }^{[8]}$. In such cases, physical properties for determining the common origin are size, shape, dimensions, thickness, colour, density, refractive index, of which the most discriminating is refractive index ${ }^{[9]}$.

Wood is composed of cellulose, lignin, hemicelluloses, and minor amounts (usually less than $10 \%$ ) of extraneous materials contained in a cellular structure $^{[10]}$. Construction of plywood is accomplished by orienting an odd number of layers with the grain direction of adjacent layers perpendicularly to one another. Fibreboard exploits the inherent strength of wood to a great extent since wood is fibrous in nature. Bonds between the wood fibres are broken to make fibre from composites, to accomplish this easily; attrition milling, or refining is used. Steaming the lignocelluloses weakens the lignin bonds between the cellulosic fibres. As a result, fibres are more readily separated and are usually less damaged than fibres processed by dry processing methods. Medium-density fibre board is frequently used in furniture applications. It is also used for interior door skins, moldings, flooring substrate, and interior trim components ${ }^{[11]}$ 
In the present study, behaviour of pellets on different targets was studied at 10 metres range which can provide aid during the investigation of crimes where projectile hits intermediary targets. The current study can be useful for determining variation in impact on different targets using same projectile. Moreover, the study can be useful in understanding the projectile behaviour can be useful in crime scene reconstruction by further using different types of projectiles.

\section{Methods}

Different materials like wood, glass, and fabrics were used as targets onto which firing was done using .177" calibre Hammerli AR 207.5 Joules where copper and lead pellets were used. The firing was carried out in controlled room at a fixed distance of 10 metres considering various factors affecting the impact. The extent of impact and damage zone were documented and analysed. The targets chosen were fabric (cotton and silk), wood (medium density fibreboard and plywood) and glass (sodalime, privacy and textured glass). The study was carried out to explore on the relationship of the entry holes produced on targets by pellets and correlate the trends and patterns in the study.

\section{FABRIC:}

$20 \mathrm{gm}$ of gelatin powder was dissolved in $80 \mathrm{cc}$ of clean water and aqueous solution was prepared. The solution was poured in the containers of size 6"X4"X4" and kept in refrigerator overnight. Temperature of $10^{\circ} \mathrm{C}$ was maintained for the gelatin block ${ }^{[12]}$. The fabric was clamped at both the ends and mounted on gelatin. The gelatin gel block was arranged suitably on a rigid platform. The gel block was supported with the base of the platform. The firearm was fired observing appropriate safety measures.

\section{FOR GLASS:}

Three types of glass namely soda lime glass, privacy pane and textured glass of $4 \mathrm{~mm}$ thickness were fired using airgun. The wooden frame was placed perpendicular to the muzzle end of the weapon used. Features such as number of radial and concentric fractures was observed. The fractured pane was measured for hole diameter, thickness and diameter of mist zone with the help of vernier caliper.

\section{Wood:}

The two types of wood used in the experiment were Plywood and Medium Density Fibreboard (MDF) 6"X6" with thickness of $4 \mathrm{~mm}$. These wooden blocks were clamped at the ends and placed on the table and kept perpendicular to the muzzle end of the firearm. The documentation was done using camera Canon EOS 200D having aperture f/5.6.

\section{Statistical design:}

Data were verified prior to computerized entry. The Statistical Package for Social Sciences (SPSS version 22.0) was used. A statistically significant difference was considered at $p$-value $p \leq 0.05$. The hypothesis framed were as follows: Null hypothesis: different targets have insignificant difference on entry hole and different pellets have insignificant difference on entry hole and exit hole.

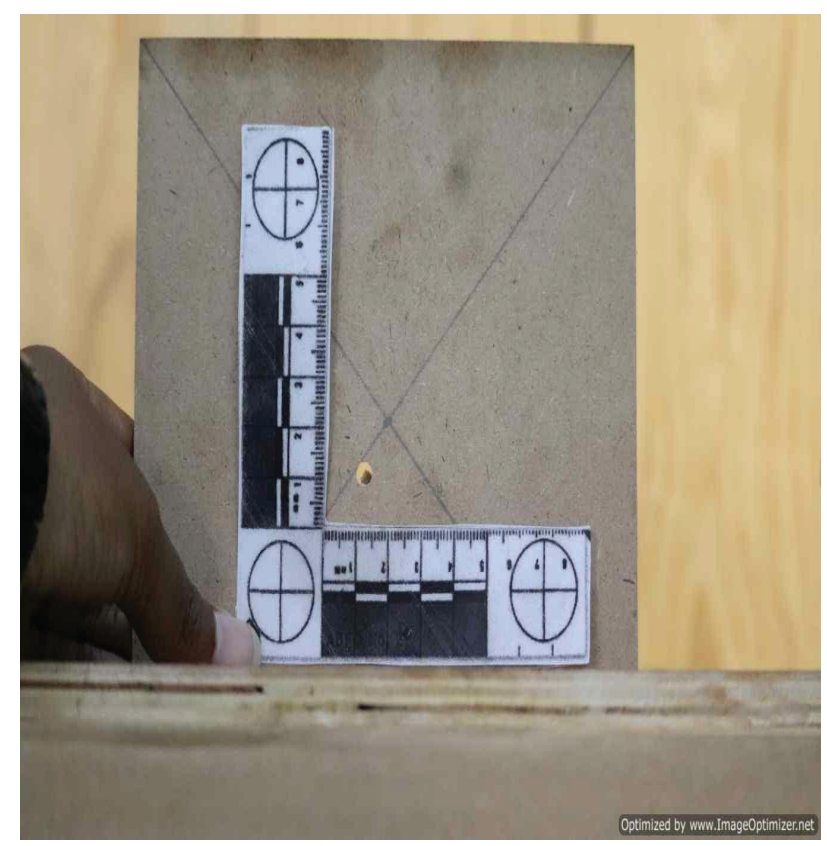

Figure 1: Impact by copper pellet on MDF 


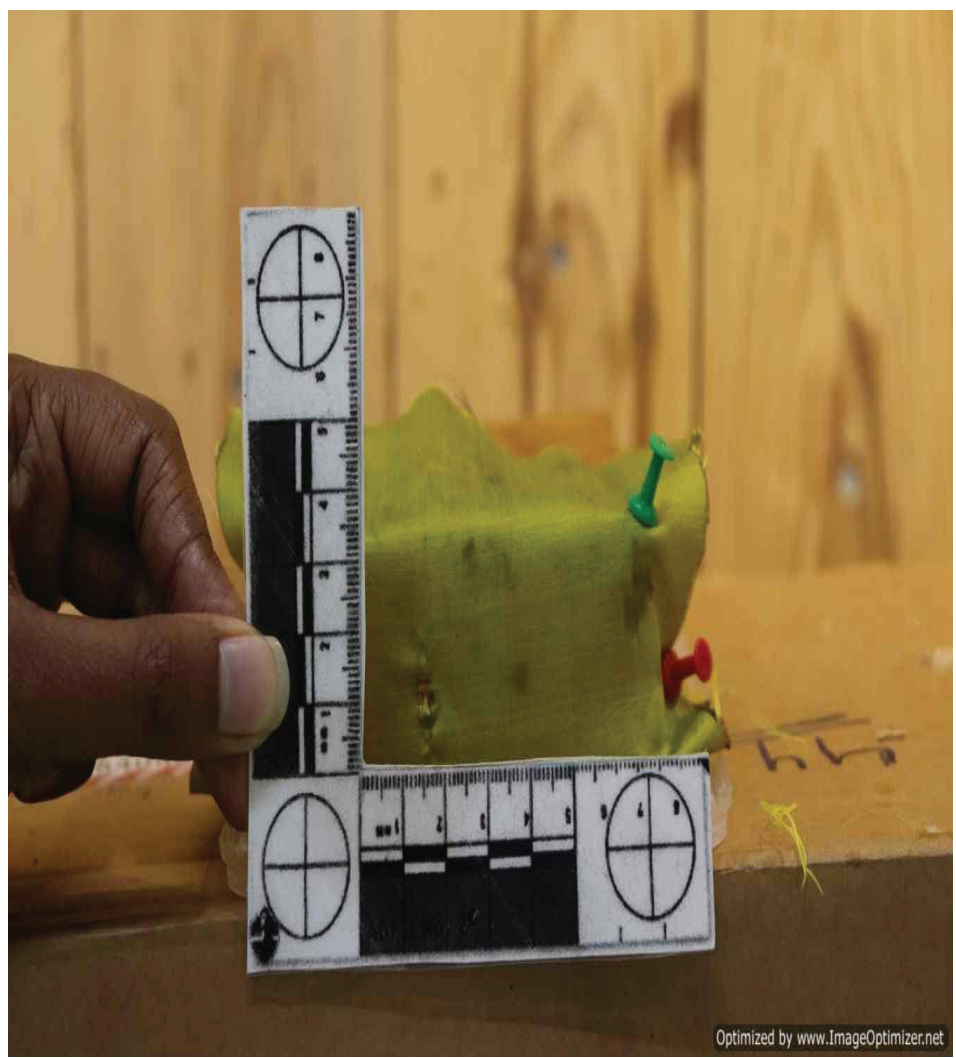

Figure 2: Impact made by copper pellet on silk

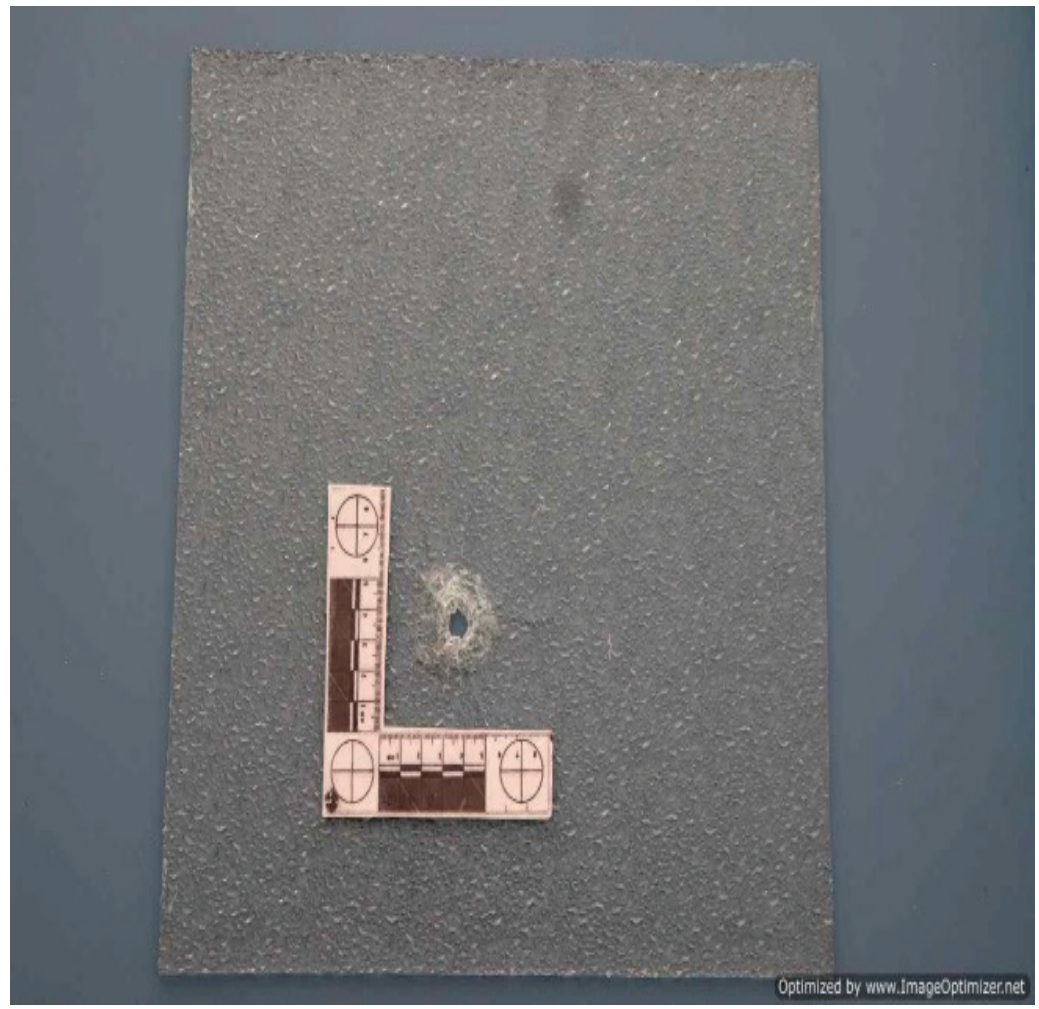

Figure 3: Impact made by Copper pellet on Textured glass 
Table 1: Mean Ranks of Different Target Groups

\begin{tabular}{|c|c|c|c|}
\hline Entry Hole & Target & $\mathbf{N}$ & Mean Rank \\
\hline \multirow{8}{*}{ Diameter of Entry Hole } & MDF & 6 & 15.75 \\
\hline & PLYWOOD & 6 & 16.33 \\
\hline & Cotton on Gelatin block & 6 & 6.00 \\
\hline & Silk on Gelatin block & 6 & 12.08 \\
\hline & Textured glass & 6 & 27.33 \\
\hline & Soda lime glass & 6 & 38.50 \\
\hline & Privacy Pane & 6 & 34.50 \\
\hline & Total & 42 & \\
\hline
\end{tabular}

Table 2: Mean Ranks of Different Pellets

\begin{tabular}{|c|c|c|c|}
\hline Entry Hole & Pellets & N & Mean Rank \\
\hline \multirow{2}{*}{ Diameter of Entry Hole } & Lead Pellet & 21 & 20.43 \\
\cline { 2 - 4 } & Copper pellet & 21 & 22.57 \\
\cline { 2 - 4 } & Total & 42 & \\
\hline
\end{tabular}

Table 3: Mean Ranks of Different Target Groups

\begin{tabular}{|c|c|c|c|}
\hline \multicolumn{4}{|c|}{ Ranks } \\
\hline Exit Hole & Target & $\mathbf{N}$ & Mean Rank \\
\hline \multirow{8}{*}{ Diameter of Exit Hole } & MDF & 6 & 23.83 \\
\hline & PLYWOOD & 6 & 3.50 \\
\hline & Cotton on Gelatin block & 6 & 11.00 \\
\hline & Silk on Gelatin block & 6 & 14.00 \\
\hline & Textured glass & 6 & 25.17 \\
\hline & Soda lime glass & 6 & 37.17 \\
\hline & Privacy Pane & 6 & 35.83 \\
\hline & Total & 42 & \\
\hline
\end{tabular}


Table 4: Mean Ranks of Different Pellets

\begin{tabular}{|c|c|c|c|}
\hline \multirow{2}{*}{ Exit Holes } & Pellets & N & Mean Rank \\
\hline \multirow{3}{*}{ Diameter of Exit Hole } & Lead Pellet & 21 & 21.74 \\
\cline { 2 - 4 } & Copper pellet & 21 & 21.26 \\
\cline { 2 - 4 } & Total & 42 & \\
\hline
\end{tabular}

\section{Discussion}

The forensic community comes across cases where the projectile is found in severely damaged condition, fragmented, or not recovered from the victim or the scene of crime. The present study was performed using different materials like wood, glass, and fabrics as targets onto which firing was done using .177" calibre Hammerli AR 20 7.5 Joules with copper and lead pellets. The firing was carried out in controlled room considering various factors affecting the impact. Figures 1,2 and 3 depict the impact made by copper pellets on MDF, silk mounted on gelatin block and textured glass, respectively. Analysis of entry and exit hole was made by studying impact on targets using Statistical Package for Social Sciences (SPSS version 22.0). Pellets perforated through the fabric of cotton and silk penetrating the gelatin block. Fibres were found to be protruded outwards around margin of entry hole whereas, when pellets perforated through wooden targets, penetration depth could not be measured.

In the current study, Kruskal Wallis $\mathrm{H}$ test, non-parametric test used to measure the significant differences was imposed. This test considered an alternate of ANOVA, as the normality and homogeneity assumption does not follow in the current dataset of entry and exit holes on all target surfaces. Table 1 represents the average of every dimension of targets on entry hole. Table 2 represents the average of pellets on entry hole. Therefore, the null hypothesis is rejected. It was found that different targets have significant difference on the entry hole whereas the pellets of same calibre used in study had insignificant difference on entry hole.

Table 3 represents the average of every dimension of target groups on exit hole. Table 4 represents the average of different pellets on exit hole. It was found that different targets have significant difference on the exit hole whereas the pellets used in study had insignificant difference on exit hole which could be possible since same calibre pellets were used for the study.

The present study can be explored further by determining variation in impact on targets using different projectiles. It can also help in understanding the projectile behaviour on the targets which helps in reconstructing the crime scene. It can further provide analytical predictions in cases where projectiles cannot be recovered, are damaged or fragmented. The fracture behaviour of the glasses upon ballistic impact can be utilized for the development of high-performance bulletproof glasses for the application in next generation automobile windshield and armours in ballistic application.

\section{Conclusion}

The forensic community comes across cases where the projectile is found in severely damaged condition, fragmented, or not recovered from the victim or the scene of crime. It becomes difficult to determine the calibre or type of projectile used in such complex scenarios. The present study was performed using plywood and MDF, sodalime, privacy pane and textured glass, and fabrics such as cotton and silk as targets using .177" calibre lead and copper pellets fired from Hammerli AR 
207.5 Joules at 10 metres. Post analysis of the present data was done to study the influence of lead and copper pellets on wood, cloth and glass on entry and exit holes. However, it was found that in both cases (i.e., entry and exit holes), the targets have a significant difference but in case of pellets of same calibre, there is an insignificant difference with entry and exit holes.

Acknowlegment: The authors would like to acknowledge Dr. Asha Rajiv (Director, School of Sciences B-II, JAIN (Deemed-to-be University), Dr. Ashwini N (Convenor, Research and Development Cell, School of Sciences B-II, JAIN (Deemed-tobe University) and Dr. Reena Susan Philip (Head, Department of Forensic Science, JAIN (Deemed-to-be University) for their constant guidance and support. The authors also would like to extend gratitude to Golden Ace Shooting Academy, Kalyan Nagar, Bangalore for their support and co-operation.

Ethical Clearance: Ethical clearance was obtained from institutional ethical board.

Funding: This research was funded by Research and Development Cell, School of Sciences B-II, JAIN (Deemed-to-be University), Bangalore, Karnataka, India.

Conflict of Interest: The authors declare that there is no conflict of interest.

\section{References}

1. Definition of Firearm. Retrieved from: https:// www.cyberabadpolice.gov.in/information/PDF/ acts-laws/Arms\%20Act.pdf

2. Reddy KS. The essentials of forensic medicine and toxicology. Journal of Punjab Academy of Forensic Medicine \& Toxicology. 2005;5(5):53-.

3. Heard BJ. Handbook of firearms and ballistics: examining and interpreting forensic evidence. John Wiley \& Sons; 2011 Aug 17.

4. Warlow T. Firearms, the law, and forensic ballistics. CRC Press; 2011 Nov 17.

5. Rosenberg Z, Dekel E. Terminal ballistics. Berlin: Springer; 2012 Mar 2.

6. Carr DJ, Stevenson T, Mahoney PF. The use of gelatine in wound ballistics research. International journal of legal medicine. 2018 Nov;132(6):165964.

7. Robertson J, Roux C, Wiggins KG. Forensic examination of fibres. CRC press; 2017 Dec 1.

8. Saferstein R. Criminalistics. Pearson Education; 2013.

9. Siegel JA, Mirakovits K. Forensic science: the basics. CRC Press; 2015 Dec 1.

10. Wiemann M. Characteristics and availability of commercially important woods. chapter 2 in FPLGTR-282. 2021:2-1.

11. Ross RJ. Wood handbook: wood as an engineering material. USDA Forest Service, Forest Products Laboratory, General Technical Report FPLGTR-190, 2010: 509 p. 1 v.. 2010;190.

12. Directorate of Forensic Science (DFS), Ministry of Home Affairs (MHA) Government of India New Delhi 2005, Laboratory Procedure Manual of Forensic Ballistics. 